\title{
Psychiatric assessment of mood instability: qualitative study of patient experience ${ }^{\dagger}$
}

\author{
Amy C. Bilderbeck, Kate E. A. Saunders, Jonathan Price and Guy M. Goodwin
}

\section{Background}

Mood instability is a common reason for psychiatric referral. very little is known about how patients with unstable mood experience assessment and diagnosis.

\section{Aims}

To investigate the experiences of assessment and diagnosis among patients with mood instability and to suggest improvements to this process.

\section{Method}

Qualitative study, gathering data through individual interviews with 28 people experiencing mood instability and receiving a psychiatric assessment in secondary care.

\section{Results}

Participants described the importance of receiving an explanation for their symptoms; the value of a good interpersonal relationship with their clinician(s); being listened to and acknowledged; and being involved in and informed about clinical decisions. These needs were not, however, consistently met. Receiving a psychiatric diagnosis, including a diagnosis of bipolar disorder or borderline personality disorder, evoked both positive and negative responses among participants, relating to stigma, personal understanding and responsibility, prognosis and treatment.

\section{Conclusions}

Patients with mood instability seek explanation for their symptoms and difficulties, empathetic care and consistent support as much as cure. Clinicians may incorrectly assume what patients' attitudes towards diagnosis are, a mismatch which may hamper the development of a strong therapeutic relationship. Clear, patient-centred communication, which acknowledges the patient's experience, may result in greater patient engagement and satisfaction.

\section{Declaration of interest}

G.M.G. received grants/research support, consulting fees and honoraria within the past 3 years from Astrazeneca, BristolMyers Squibb, Cephalon/Teva, Eli Lilly, Lundbeck, Otsuka, P1Vital, Roche, Servier, Sunovion and Takeda.
Mood instability has a relatively high prevalence in the general population (estimated at $13.9 \%)^{1}$ and is a common reason for referral to adult community mental health teams (CMHTs) with up to $80 \%$ of patients reporting this symptom at assessment. ${ }^{2}$ Very little is known about the experience of assessment within this patient group. There has been some limited research into the experiences of patients receiving one of the two common differential diagnoses in this group, namely bipolar disorder and borderline personality disorder. ${ }^{3-5}$ However, this previous work has focused on the experience of receiving a diagnosis and treatment services, rather than the experience of assessment per se. Moreover, by no means all patients who are assessed will receive a diagnosis. Understanding more about the patient experience of assessment in secondary psychiatric care could enhance communication between clinicians and patients, inform training and facilitate the diagnostic process. Given the exploratory nature of this research, we adopted a qualitative design and 'framework' analysis approach, which has been specifically developed for applied or policy-relevant qualitative research. ${ }^{6,7}$

\section{Method}

\section{Study design}

This study used patient interview data gathered as part of a project that also involved the observation of assessments and interviews with clinical staff. Ethical approval for the study was obtained from Oxfordshire Research Ethics Committee A (11/H0604/8).

\section{Participants}

Participants were patients referred by their general practitioners (GPs) to secondary mental healthcare teams in Oxfordshire and

'See editorial, pp. 178-179, this issue.
Buckinghamshire. For all participants, mood instability had been cited as a reason for referral. We recruited patients from seven CMHTs, a specialist mood disorders clinic and a complex needs service (an out-patient therapeutic community). Other inclusion criteria included being 18 years old or over, and fluent in spoken and written English. Purposive sampling was used to ensure variation in gender, age, previous diagnoses and treatment regimes, and clinical setting at the point of recruitment. One patient declined to participate without giving a reason, and one consented to the researcher being present in their assessment, but then withdrew from the study and was not interviewed. No other patients who were approached declined to participate. Written, informed consent was obtained from all participants, and also from patients' assessing clinicians.

\section{Data gathering}

Basic demographic data and medical records data were gathered for all participants.

\section{Interviews}

Where possible, the clinical assessment was observed by A.C.B. before the qualitative interview was conducted (22 participants, 78.6\%). Qualitative semi-structured interviews were conducted by A.C.B. and K.E.A.S., as part of an iterative process of data collection and analysis. Interviews were audiorecorded, with the permission of the participant, and lasted between 20 and 70 min. Our topic schedule is in the online supplement DS1. Participants were asked to describe their general experience of assessment, and to comment specifically on their expectations, suggestions for improvement, feelings about the outcomes of their assessment, and any other issues. As the study progressed, and understanding developed, we altered the topic schedule to focus 
the interview on emerging themes. We allowed the data itself to drive the development and inclusion of new questions. For example, participants spontaneously spoke about their expectations prior to assessment, leading us to include the question 'Did you have any expectations of the assessment?'. Because these questions were derived from the interviews themselves, we do not feel that they resulted in substantial bias in the data collected. Potential changes to the topic schedule were discussed among members of the research team at regular meetings and agreed by consensus. Questions remained broad and open, to promote the emergence of further themes. Potential insights gained through observation of the assessment, such as salient points raised or discussions between the patient and clinician about potential diagnoses, also guided interviewing. Participants who completed multiple diagnostic assessments were invited to take part in multiple interviews in order to track their experience.

Clearly, patients' views of their clinical assessment might change with time and, especially, as they engaged with and perhaps benefited from planned treatments. We therefore also invited participants to take part in a follow-up interview, 6 months later, to better understand their longer-term perceptions of the assessment and diagnosis. Data gathering ceased when understanding was no longer being advanced, i.e. at the point of data saturation (see below).

\section{Data analysis}

Audiotaped interviews were transcribed by A.C.B., K.E.A.S. or an experienced transcriber, reviewed by A.B., and uploaded to proprietary qualitative analysis software, NVivo 9 on Windows (QSR International Pty Ltd, Doncaster, Victoria, Australia, www.qsrinternational.com). The qualitative analysis used a simple framework technique, which is described in detail by Ritchie \& Spencer. ${ }^{6}$ In brief, the first few transcriptions were read and re-read as open-mindedly as possible (our methods of reducing bias in data interpretation are described below), and then phrases were coded in an initial round of analysis. Coding comprised identification of attitudes or experiences, referred to as 'themes', described in participant interviews. NVivo analysis software also enabled, at any time, review of the frequency with which themes were raised across participants, as well as demographic and clinical information that described the participants contributing to each theme. Opinions on the meaning and significance of specific phrases were discussed between researchers. As understanding increased, and through constant comparison of new data with the emerging understanding, data were interpreted and summarised, new codes generated, redundant codes deleted and overlapping codes merged. Steadily, a stable framework of specific codes emerged that appeared increasingly likely to describe participant experiences of assessment. A.C.B. conducted the data analysis, K.E.A.S. co-analysed some of the data. The study team met regularly to discuss findings and refine the emerging understanding.

A key challenge for any qualitative study is to maximise rigour and minimise bias. Our research team consciously included a primary data gatherer and analyst with a non-medical background (A.B.). The researchers discussed and maintained an awareness of preconceptions (facilitated by note-keeping and memos) and the ways in which these could influence recruitment, data gathering and data analysis. Strenuous and repeated efforts were made to 'ground' the emerging understanding in the participant data. Quantitative data were summarised using standard statistical approaches.

\section{Results}

Table 1 summarises the demographic and clinical characteristics of the 28 participants. Twenty-seven participants (96\%) had received an assessment within the past month, and assessments for 22 participants (79\%) were observed prior to interviewing. The assessing clinicians included eight psychiatric consultants, four core trainees, three psychiatric nurses, two associate specialists, two specialist trainees and one foundation doctor. Four participants completed two post-assessment interviews and one participant completed three post-assessment interviews. Additionally, 11 participants completed a 6-month follow-up interview, resulting in an overall total of 45 research interviews.

\section{Conventions}

Quotations are identified by: a consecutive study number; gender $(\mathrm{M} / \mathrm{F})$; and diagnosis. We use the following key to indicate diagnosis: $\mathrm{BD}$, bipolar disorder (type II for all participants); $\mathrm{BPD}$, borderline personality disorder; $\mathrm{PD}$, other or unspecified personality disorder; DA, depression and/or anxiety; ND, no or unknown diagnosis; OD, other diagnosis. So, for example (P06-F-BD) is participant number 6, female, diagnosed with bipolar II disorder.

In describing the results of our analysis, we have adopted specific terms that indicate how frequently participants discussed certain themes. These are: 'some' $(n=3-7,11-25 \%)$, 'many' $(n=8-14$; more than $25 \%$, up to $50 \%)$ and 'most' $(n=15-28$, more than $50 \%$ ).

Participants discussed many issues but they could be captured within five broad themes: (a) wanting an explanation and help; (b) wanting consistent and continuous care; (c) struggling to communicate and be understood; (d) wanting to feel involved and informed; and (e) wanting to be acknowledged, but often

Table 1 Participants' demographic and clinical characteristics

\begin{tabular}{|c|c|}
\hline & $\begin{array}{l}\text { Participants } \\
\qquad(n=28)\end{array}$ \\
\hline Age, years: mean (s.d.) range & 36 (12) 20-58 \\
\hline Female: $n(\%)$ & $18(64)$ \\
\hline \multicolumn{2}{|l|}{ Employment, ${ }^{a} n(\%)$} \\
\hline Employed, full/part-time & $7(26)$ \\
\hline Unemployed/DLA or incapacity benefit & $16(59)$ \\
\hline Student & $4(15)$ \\
\hline \multicolumn{2}{|l|}{ Marital status, ${ }^{a} n(\%)$} \\
\hline Married/cohabiting & $10(37)$ \\
\hline Divorced/separated & $5(19)$ \\
\hline Single & $11(41)$ \\
\hline Widowed & $1(4)$ \\
\hline \multicolumn{2}{|l|}{ Ethnicity, ${ }^{a} n(\%)$} \\
\hline British, White & $24(89)$ \\
\hline White, other & $2(7)$ \\
\hline British, mixed & $1(4)$ \\
\hline \multicolumn{2}{|l|}{ Location of recruitment, $n$ (\%) } \\
\hline Community mental health teams & $15(54)$ \\
\hline Specialist mood disorders clinic & $8(29)$ \\
\hline Complex needs service & $4(14)$ \\
\hline Psychological services & $1(4)$ \\
\hline \multicolumn{2}{|l|}{ Diagnosis received, ${ }^{\mathrm{b}} \mathrm{n}(\%)$} \\
\hline Bipolar II disorder & $5(18)$ \\
\hline Borderline personality disorder & $3(11)$ \\
\hline Other personality disorder & $2(7)$ \\
\hline $\begin{array}{l}\text { Comorbid bipolar disorder (previous diagnosis } \\
\text { of borderline personality disorder) }\end{array}$ & $1(4)$ \\
\hline Depression/anxiety & $8(29)$ \\
\hline Other diagnosis & $2(7)$ \\
\hline No diagnosis or unknown & $7(25)$ \\
\hline \multicolumn{2}{|l|}{$\begin{array}{l}\text { DLA, disability living allowance. } \\
\text { a. Demographic data were not collected from one participant. } \\
\text { b. As described in assessment letters. }\end{array}$} \\
\hline
\end{tabular}


feeling dismissed and discredited. These themes are described and expanded on below. Key quotations are embedded within the main text; additional quotations are provided in online supplement DS2. As the themes emerging from initial and follow-up interviews were very similar, the entire data-set was ultimately combined into a single analysis. One unique subtheme that arose from follow-up interviews, relating to patients' desire for reliable and consistent support after assessment, is described within the 'Wanting consistent and continuous care' theme. Themes did not differ with gender or diagnosis, and this is reflected in the supporting quotations reported here and in online supplement DS2, which show a variety of participant characteristics within each theme.

\section{Wanting an explanation and help}

Participants frequently described attending their assessments with the hope that it would provide them with an explanation for the difficulties they were experiencing, and offer practical help in dealing with difficult patterns of mood and behaviour. However, some qualified their hopes by feeling sceptical about whether their assessment would result in meaningful help, and had limited expectations of care.

'I mean she might be my saviour I don't know ... you know after 30 years - it's probably longer than that - um, you get used to it . . . What I actually expected was ... the usual stuff and then she'd prescribe a different drug.' (P20-M-PD)

Many participants felt that a primary purpose of their assessment was to rule in, or rule out, a specific diagnosis of bipolar disorder. However, some participants expressed how obtaining an explanation of their symptoms and effective help was more important to them than receiving any specific diagnosis.

'He knew I had gone there to find out if I was bipolar full stop and if I wasn't . . . so why was I acting so out of character for so many weeks of the year?' (P07-F-BD-BPD)

By providing some explanation many participants described how receiving a diagnosis, including a diagnosis of bipolar or borderline personality disorder, could give them a sense of containment, validation and recognition. In many cases the explanation provided by diagnosis was also seen to remove a sense of personal responsibility and blame for previous harmful behaviours. This could be the case for participants receiving a personality disorder diagnosis or a diagnosis of bipolar disorder.

'It's [a diagnosis of bipolar disorder] taking a bit of the guilt away that I feel for all the things that have happened, that I've done ...' (P07-F-BD-BPD)

'To actually like know that it's not my fault I'm not a complete nutter ... [it's] sort of almost just like a relief really.' (P03-F-BPD)

Three participants expressed relief at not receiving a diagnosis of bipolar disorder. This was linked, for each of these participants, to the perceived negative implications of receiving the diagnosis (such as stigma, apprehensions about taking medication). In addition, two of these participants felt that a diagnosis of bipolar disorder did not adequately describe the problems they experienced.

'[l'm] very pleased ... for me it's the stigma that's attached to it . . . I felt that I didn't have bipolar.' (P16-F-DA)

\section{Wanting consistent and continuous care}

Frequently participants emphasised the importance of continuity of care. Continuity of care and familiarity with clinician(s) was linked with more personal and meaningful discussions during the assessment, reduced anxiety and greater comfort in discussing potentially sensitive life histories. Conversely, meeting with unfamiliar clinicians was linked to assessments that were less personal and could prompt anxiety. Some participants felt frustrated with the lack of continuity of care they received.
'There's never any consistency. It really bothered me ... Virtually a different doctor every time I went back ... it's just like going back to a stranger and I don't really, it's not helping me at all.' (P20-M-PD)

Continuity of care was also seen to be important because it reduced the need to repeat (often painful) life histories. A few participants expressed frustration in cases where they felt that sensitive information about life history had already been gathered in previous assessments.

'I'd like to see the same doctor all the time. See one, than have to explain myself again and again and again.' (P12-M-ND)

Feeling supported in the period of time after assessment was also important to participants, and frequently discussed in follow-up interviews. However, some participants did not feel well supported in the months following assessment and diagnosis.

'They're supposed to see you every one to two weeks just to see that the medication's going well. Um, and of course that wasn't the case ... these are um numbers in case you need to speak to somebody, um you know you're not on your own etcetera etcetera, that should, I think, should all be given and spoken about at the beginning.' (P28-F-BD)

\section{Struggling to communicate and be understood}

Participants identified several barriers to effective communication with their clinicians. Participants felt considerable pressure to answer clinicians' questions accurately, and many felt that they had failed to do this.

'I just say things off the top of my head ... I've probably given some wrong answers there.' (P11-F-ND)

Difficulty recalling relevant information was one reason for this. Participants could also be critical of the need to answer clinicians' questions fully within the time constraints of the assessment.

'They expect you to be able to just give them a nice little synopsis ... it's not gonna happen.' (P04-F-BPD).

Many participants described the challenge of communicating hidden, subjective mood states. Some participants expressed frustration with the need to objectively and accurately answer clinicians' seemingly ambiguous questions.

'Being asked "have you ever had times where you felt much better than usual", what does that mean? Because everybody's had times where they've felt much better than usual.' (P06-F-BD)

Some participants, who received a diagnosis of bipolar disorder, reflected that hypomanic episodes had not been identified during previous assessments because their own help-seeking behaviour had primarily centred on ameliorating depression and anxiety, and this had influenced both the focus of assessments and the clinician's understanding.

'I always used to go and see them when I was low ... No-one ever talked about the highs and things like this, it was always the lows.' (P28-F-BD)

Many participants felt that family and friends could facilitate communication with clinicians. Follow-up assessments were also seen as a way of improving clinicians' understanding of participants' difficulties, again emphasising the importance participants attributed to continuity of care.

Some participants described the significance of difficult or abusive early life experiences in understanding the problems they currently experienced. However, communicating with clinicians about those events could be distressing. Participants who found talking about their past distressing would often try to avoid expanding on these topics, and appreciated clinicians who were sensitive to this. However, some participants felt that clinicians placed too great an emphasis on information about previous trauma, at the expense of exploring more recent problems.

'I just thought she [previous clinician] is just blaming it on my dad, such a bastard, and my mum was hopeless and that's why you are like this today and we'll put it down to personality disorder, whereas he [current clinician] went into a lot more things that are actually relevant as in who I am and how I behave.' (P07-F-BD-BPD) 


\section{Wanting to feel involved and informed}

Participants expressed the need to feel informed by their clinicians about their diagnosis, and involved in decisions about treatment. 'It was quite nice to like for him to say I don't think it's bipolar, because - this reason, this reason, this one. But, I do think you are a little bit borderline because of this, this and this ... He was like explaining it in a like a quite a simple way sort of thing instead of like "you're this and that's it".' (PO3-F-BPD)

Unfortunately, in many cases participants did not feel informed or involved.

'It would have been helpful to come away with some information ... it was only when I was actually in the waiting room that I picked up some leaflets.' (P02-F-BD)

Participants in many instances described anxiety about perceived stigma surrounding diagnosis, including diagnoses of both bipolar and borderline personality disorder.

'In my eyes, there's that big question of what's normal, depression is normal, it's that, bipolar ... ain't normal.' (P09-M-DA)

'It [a diagnosis of BPD] makes it sound like it's my fault, it's my personality . . [like] I was born wrong so it was never going to be good.' (P04-F-BPD)

Some participants described how they felt that clinicians had failed to discuss the sources of stigma with them.

'Nobody told me what bipolar was or anything like this so I had to do a lot of research online ... Why, why is this happening to me? What's caused it?....and I don't think I was given any um indication or, or whatever.' (P28-F-BD)

\section{Wanting to be acknowledged, but often feeling dismissed and discredited}

Frequently participants described how feeling listened to, empathised with and cared about was important in their assessment. Participants appreciated clinicians who gave them time to speak about their problems, and this process was seen as beneficial in itself.

However, participants also frequently described feeling abandoned and dismissed in recent or previous assessments. The reasons for this were diverse. Some felt that a lack of time caused consultations to be rushed and formulaic. Assessments that seemed hurried, overly formal or impersonal, and clinicians who it was felt did not acknowledge their client's suffering, left participants feeling frustrated and unheard. Some participants felt that clinicians distrusted them and discredited their accounts of the severity of their problems because these were inconsistent with their behaviour and personal presentation in assessment.

'Because I'm not waving a knife and saying I'm going to kill myself, they're like, well you're not quite, you're not quite sick enough ... I'm trying my hardest to tell them and explain ...' (P04-F-BPD)

Many participants felt that their interactions with clinicians and clinical teams could be described as 'a vicious circle' (P20-M-PD) or '[going] pillar to post' (P10-M-ND, P18-F-ND, P04-F-BPD). This experience was frequently associated with descriptions of inconsistent care, changing diagnoses, polypharmacy and ineffective treatments. Many participants were dismayed by perceived inflexibility in the treatment options that their clinicians offered them or described feeling 'fobbed off' by medication, and thereby denied a more thoughtful appraisal of their problems.

Some participants felt abandoned by mental health services in part because they had not received a diagnosis. One participant felt uncertain about whether she had a diagnosis of borderline personality disorder and, associated with this, expressed feeling rejected.

I'm still not even sure if I have a diagnosis of borderline personality disorder even though they're asking me to attend these [Complex Needs] groups so yeah I'm confused and not particularly happy ... you do feel like you're being palmed off without any diagnosis at all.' (P04-F-BPD)

Another participant described frustration when they felt that the diagnosis of borderline personality disorder had been concealed. 'No one ever mentioned PD ... it felt like it had been kept a secret.' (P04-F-BPD).
However, in several cases participants felt that being given a diagnosis could be dismissive in itself. This could apply to different diagnoses, including diagnoses of bipolar or borderline personality disorder, and was linked to a perceived lack of support, information and explanation, which led participants to question the utility of diagnosis.

'It was like I was told I had the disorder, these are your tablets, and we'll see you next week ... a lot more information could have been given ... just more explanation ... it actually makes you feel a little bit worse, because yes you've been told you've got this bipolar, um, go on and get on with it ... ' (P28-F-BD)

One participant questioned the utility and meaningfulness of a personality disorder diagnosis, which was seen to be impersonal and dismissive in itself.

'A proper diagnosis um that doesn't say I have personality disorder would be really nice.... it feels like the easy way out .... I think it's just the cheap way to treat lots of people ... . something to put on the doctor's notes . . . it's not personal at all.' (P04-F-BPD)

\section{Discussion}

\section{Principal findings}

We found that individuals referred for psychiatric assessment of mood instability placed a primary emphasis on finding an explanation for their problems. This view was expressed irrespective of the subsequent diagnosis they received. The need for a greater understanding of presenting problems has been described in a number of patient groups including those with medically unexplained physical symptoms ${ }^{8}$ and patients attending GP surgeries. ${ }^{9}$ Participants were frequently ambivalent about receiving a diagnosis of, for example, bipolar or borderline personality disorder. Some participants expressed shock and/or fear on receipt of a formal diagnosis, but for many, diagnosis was helpful and contributed to a meaningful explanation of their symptoms. Many participants felt the receipt of a diagnosis absolved them from feeling excessively responsible for their problems. Personality disorder diagnoses have been perceived by patients and clinicians as particularly denigrating and stigmatising ${ }^{3,4,10}$ but we found no difference between those given a borderline personality disorder diagnosis and those given a bipolar disorder diagnosis. Diagnosis was experienced by some participants as dismissive, in a way that was linked to a perceived lack of adequate explanation and/or support. Similar experiences are reported by those with somatisation disorders. ${ }^{11,12}$

Participants emphasised the value they placed on a good professional relationship with their clinicians, as well as needing to feel listened to and empathised with, consistent with findings in many patient groups ${ }^{13-15}$ including patients diagnosed with bipolar disorder. ${ }^{16}$ Several specific barriers to communication were also identified: participants reported feeling anxiety and pressure to give the 'correct answers' in assessment; experienced difficulties with recall; and acknowledged the challenge of communicating subjective states. Notably, many participants described feeling the need to convince clinicians of the legitimacy of symptoms, similar to the experiences of patients with conditions associated with medically unexplained symptoms. ${ }^{17}$

\section{Strengths and limitations}

This is the first study to explore the experiences and expectations of patients with mood instability undergoing psychiatric assessment. By enhancing our understanding of patient perspectives, this study highlights how psychiatric assessment might be improved in order to better meet patients' needs. This is particularly important, given the difficulties in discerning the differential diagnosis in those with mood instability and the subsequent impact diagnosis can have on treatment. 
Our interview schedule was regularly revised based on data gathered, allowing an in-depth examination of relevant issues. By interviewing most participants shortly after observation of clinical assessments, we were able to explore patient attitudes and responses that are a possible indicator of later engagement. ${ }^{18}$ Additional 6-month follow-up interviews provided the opportunity to further explore attitudes towards personality disorder diagnoses, and although we found no evidence for altered attitudes towards diagnosis at 6 months, it remains possible that perceptions, and in particular increased perceptions of stigma, may change over longer periods of time. ${ }^{4,19}$

The diversity of attitudes, experiences and backgrounds of our participants make it likely that the issues identified here are relevant beyond the context of this research. However, although researchers emphasised their neutrality, their separation from the clinical team may have resulted in self-selection of participants into the study. Previous displeasure with services, psychopathology and socially desirable responding in interviews could have modified views expressed by the participants. Purposive sampling allowed minimisation of these sources of bias. Finally, patients' and clinicians' knowledge of research participation, and in particular observation of assessments, may have influenced the interaction between clinicians and participants. However, observation of assessments enabled researchers to tailor interviews more closely to individuals' experience, focusing on specific details of the assessment process, around which recommendations for practise can be formed.

\section{Implications}

The themes emerging from this analysis are almost universally described for patients presenting to doctors with complex problems not easily encapsulated by a simple diagnosis of strong validity. Clinicians should pay greater attention to patient expectations in the context of psychiatric assessment rather than assume that diagnosis is the primary outcome being sought. Clinicians may be able to improve patient experience of their assessments by acknowledging these expectations and needs. A greater focus on the patient's goals should enhance the therapeutic relationship and minimise patient disenfranchisement. Communication strategies developed in the context of managing patients with unexplained medical symptoms, for example in the promotion of explanations that are grounded in, and address, patients' concerns, ${ }^{20,21}$ may provide some guiding principles.

Patients vary in how they value a diagnosis as an explanation of a pattern of symptoms or behaviours. The decision by a clinician to deliver a diagnosis involves consideration of a number of factors, including the risk of compromising patient engagement, ${ }^{22}$ perceived stigma ${ }^{23}$ and utility. However, we found many instances where the delivery of either a bipolar disorder or a borderline personality disorder diagnosis was experienced positively, and at times was seen to help them see themselves as unwell rather than 'bad'; it thereby provided relief from guilt about past behaviours.

It has been suggested that clinicians can find it difficult to empathise with people experiencing mood instability, ${ }^{24}$ perhaps in part because of perceived diagnostic uncertainty, and other challenges in managing this patient group. ${ }^{10,25,26}$ This combination of factors may have contributed to the feelings of being distrusted and discredited that some participants described in this study. Highlighting the ambiguous nature of subjective states might be one way that clinicians can reassure patients of the legitimacy of their experience. Our findings also suggest how involvement of family and friends in assessments can help clarify symptoms and presenting problems and thereby increase patient confidence in feeling heard.

The themes we identified saturated in the present sample with participant numbers comparable with similar studies. ${ }^{4,13}$ Such results are inherently exploratory but generate the testable hypothesis that similar experiences and attitudes are likely to be evident in larger patient groups experiencing mood instability. There is a need for further research into how the assessment experience might be improved to ensure that patients' expectations are better accommodated, as well as the views of clinicians conducting assessments. Our use of a presenting symptom - mood instability - rather than a post hoc diagnosis to identify patients also adds generalisability, and we feel highlights the need for more research that takes a transdiagnostic approach in order to explore the experience of patients with diffuse presenting symptoms.

Amy C. Bilderbeck, BA, MA, DPhil, Kate E. A. Saunders, BM, BCh, MA, MRCPsych, Jonathan Price, DPhil, MRCPsych, Guy M. Goodwin, FMedSci, Department of Psychiatry, University of Oxford, Warneford Hospital, Oxford, UK

Correspondence: Jonathan Price, Department of Psychiatry, Warneford Hospital, University of Oxford, Oxford OX4 7JX, UK. Email: jonathan.price@psych.ox.ac.uk

First received 26 Feb 2013, final revision 14 Oct 2013, accepted 25 Oct 2013

\section{Funding}

This report is independent research commissioned by the NHS Research for Patient Benefit Programme (PG-PB-0909-19070).

\section{Acknowledgements}

We thank the participants who generously gave their time, and who often spoke about difficult personal issues, in order to contribute to this research. Similarly, we thank the clinical staff who contributed their time and expertise, and to those members of staff who helped recruit participants for the study.

\section{References}

1 Marwaha S, Parsons N, Flanagan S, Broome M. The prevalence and clinical associations of mood instability in adults living in England: results from the Adult Psychiatric Morbidity Survey 2007. Psychiatry Res 2013; 205: 262-8.

2 Gilbert P, Allan S, Nicholls W, Olsen K. The assessment of psychological symptoms of patients referred to community mental health teams: distress, chronicity and life interference. Clin Psychol Psychother 2005; 12: 10-27.

3 Horn N, Johnstone L, Brooke S. Some service user perspectives on the diagnosis of borderline personality disorder. J Ment Health 2007; 16: 255-69.

4 Nehls N. Borderline personality disorder: the voice of patients. Res Nurs Health 1999; 22: 285-93.

5 Proudfoot JG, Parker GB, Benoit M, Manicavasagar V, Smith M, Mcrim AG. What happens after diagnosis? Understanding the experiences of patients with newly-diagnosed bipolar disorder. Health Expect 2009; 12: 120-9.

6 Ritchie J, Spencer L. Qualitative data analysis for applied policy research. In Analyzing Qualitative Data (eds A Bryman, R Burgess): 173-94. Routledge, 1994.

7 Pope C, Ziebland S, Mays N. Qualitative research in health care - analysing qualitative data. BMJ 2000; 320: 114-6.

8 Salmon P, Dowrick CF, Ring A, Humphris GM. Voiced but unheard agendas: qualitative analysis of the psychosocial cues that patients with unexplained symptoms present to general practitioners. Br J Gen Pract 2004; 54: 171-6.

9 Little P, Everitt H, Williamson I, Warner G, Moore M, Gould C, et al. Preferences of patients for patient centred approach to consultation in primary care: observational study. BMJ 2001; 322: 468-72.

10 Deans $\mathrm{C}$, Meocevic E. Attitudes of registered psychiatric nurses towards patients diagnosed with borderline personality disorder. Contemp Nurse 2006; 21: 43-9.

11 Salmon P, Peters S, Stanley I. Patients' perceptions of medical explanations for somatisation disorders: qualitative analysis. BMJ 1999; 318: 372-6. 
12 McWhinney IR, Epstein RM, Freeman TR. Rethinking somatization. Ann Intern Med 1997; 126: 747-50.

13 Price J, Farmer G, Harris J, Hope T, Kennedy S, Mayou R. Attitudes of women with chronic pelvic pain to the gynaecological consultation: a qualitative study. BJOG 2006; 113: 446-52.

14 Heijmans M, Hartman TCO, van Weel-Baumgarten E, Dowrick C, Lucassen PLBJ, van Weel C. Experts' opinions on the management of medically unexplained symptoms in primary care. A qualitative analysis of narrative reviews and scientific editorials. Fam Pract 2011; 28: 444-55.

15 McCabe R, Roder-Wanner UU, Hoffmann K, Priebe S. Therapeutic relationships and quality of life: association of two subjective constructs in schizophrenia patients. Int J Soc Psychiatry 1999; 45: 276-83.

16 Highet NJ, McNair BG, Thompson M, Davenport TA, Hickie IB. Experience with treatment services for people with bipolar disorder. Med J Aust 2004; 181: S47-51.

17 Page LA, Wessely S. Medically unexplained symptoms: exacerbating factors in the doctor-patient encounter. J R SOC Med 2003; 96: 223-7.

18 Ware Jr JE, Davies AR. Behavioral consequences of consumer dissatisfaction with medical care. Eval Program Plan 1983; 6: 291-7.

19 Rusch N, Holzer A, Hermann C, Schramm E, Jacob GA, Bohus M, et al. Self-stigma in women with borderline personality disorder and women with social phobia. J Nerv Ment Dis 2006; 194: 766-73.
20 Rosendal M, Fink P. Management of medically unexplained symptoms includes diagnosis, specific treatments, and appropriate communication BMJ 2005; 330: 4-5.

21 Aiarzaguena JM, Grandes G, Gaminde I, Salazar A, Sanchez A, Arino J. A randomized controlled clinical trial of a psychosocial and communication intervention carried out by GPS for patients with medically unexplained symptoms. Psychol Med 2007; 37: 283-94.

22 Lequesne ER, Hersh RG. Disclosure of a diagnosis of borderline personality disorder. J Psychiatr Pract 2004; 10: 170-6.

23 Sanislow CA, Marcus KL, Reagan EM. Long-term outcomes in borderline psychopathology: old assumptions, current findings, and new directions. Curr Psychiatry Rep 2012; 14: 54-61.

24 Woollaston K, Hixenbaugh P. 'Destructive Whirlwind': nurses' perceptions of patients diagnosed with borderline personality disorder. J Psychiatr Ment Health Nurs 2008; 15: 703-9.

25 Kealy D, Ogrodniczuk JS. Marginalization of borderline personality disorder J Psychiatr Pract 2010; 16: 145-54.

26 Hersh R. Confronting myths and stereotypes about borderline personality disorder. Soc Work Ment Health 2008; 6: 13-32.

\section{psychiatry in literature}

\section{Not I: Beckett and psychiatry}

\author{
Elizabeth Barry, Matthew Broome, Jonathan Heron
}

On 23 September 1935, Samuel Beckett wrote to his friend Thomas MacGreevy: 'I went down to Bedlam this day week, and went round the wards for the first time, with scarcely any sense of horror, though I saw everything, from mild depression to profound dementia' (The Letters of Samuel Beckett, Vol I, p. Xx). Beckett was, in this visit, following a long tradition of writers visiting the Bethlem Royal Hospital in search of creative inspiration. A fictitious London asylum (the Magdalen Mercyseat) and its patients (including, in Beckett's words, those 'melancholic ... hebephrenic ... hypomanic ... schizoid') duly appear in Beckett's comic novel, Murphy (1938). Beckett's interest in mental disorder was, however, deeper than such early characterisations might suggest. His concerns about his own anxiety, depression and psychosomatic illness prompted him to undertake psychoanalysis with Wilfred Bion at the Tavistock Clinic, and his work offers increasingly sensitive representations of mental distress and speech disorder. His play Not I (1972), for instance, features a disembodied (but female) mouth speaking in fractured phrases about a past traumatic event, possibly a rape. Across the stage, a hooded Auditor listens silently, responding only to lift his arms in a gesture of 'helpless compassion'. The character ('Mouth') describes the shift from a state of near mutism ('not as much as goodbye') to one characterised by a pathological pressure of speech, creating acute social anxiety. This tachylalia is enacted by Mouth, although she refuses to identify herself with the subject of her tale ('she found herself in the ... what? who? ... no! ... she!').

These very words are scrawled on the personal script of Billie Whitelaw, the performer directed in the role of 'Mouth' by Anthony Page (and Samuel Beckett) in 1973. Whitelaw's experiences of performing the play were combined with new approaches to staging Beckett in an event, Beckett and Brain Science, held at the University of Warwick in collaboration with London (Birkbeck) and Reading in 2012, which brought together humanities scholars, arts practitioners, psychiatrists and trainee doctors. The theatre company Fail Better Productions worked with clinicians to investigate practically how Beckett's characters might inform clinical care.

The trainee doctors found the performance work particularly interesting in relation to the interplay between Mouth and the silent Auditor. We conducted some exploratory activities between these two characters and their analogous relationship to the roles of patient and clinician. Her 'whole body like gone ... just the mouth . . . like maddened . . . and can't stop . . . no stopping it' became an embodiment of anxiety and pressured speech which the workshop participants could safely explore through both the Auditor's 'helpless compassion' and the experience of speaking the rapid monologue. This workshop, which has now been adapted for NHS Kent, Surrey and Sussex Deanery, and delivered to a group including consultant psychiatrists, specialist nurses and geriatricians, offers medical educators a way to use Beckett in their classroom, and to explore problems relevant to both compassion and care in practice. The play becomes a phenomenological case history and the methods of the workshop offer strategies for interpersonal treatment. 